\title{
Force Control for Active Chassis Balancing
}

\section{Journal Article}

Author(s):

Hutter, Marco (D); Leemann, Philipp; Hottiger, Gabriel; Figi, Ruedi; Tagmann, Stefan; Rey, Gonzalo; Small, George

Publication date:

2017-04

Permanent link:

https://doi.org/10.3929/ethz-a-010723165

Rights / license:

In Copyright - Non-Commercial Use Permitted

Originally published in:

IEEE/ASME Transactions on Mechatronics 22(2), https://doi.org/10.1109/TMECH.2016.2612722 


\title{
Force Control for Active Chassis Balancing
}

\author{
Marco Hutter, Philipp Leemann, Gabriel Hottiger ${ }^{1}$, Ruedi Figi, Stefan Tagmann ${ }^{2}$, Gonzalo Rey and George Small ${ }^{3}$
}

\begin{abstract}
This paper presents the realization of the worldwide first automated walking excavator chassis. To this end, the authors build a new generation of high-performance hydraulic valves with integrated pressure feedback to achieve fast and accurate cylinder force tracking. This allows to automatically adapt the legs to uneven terrain and to optimally shape the ground reaction forces in order to change orientation and height of the cabin. Due to the contact redundancy, automated balancing is implemented as a contact force optimization problem including constraints on contact forces and joint torques. The corresponding prioritized optimization problem can be simplified by using a quasi-static approximation of the system dynamics and a complexity reduction due to the kinematic structure of the legs. Our approach considers the unknown configuration and load of the cabin, arm, and bucket as system disturbances, whereby gravitational effects are approximated as well as possible. It is tested in a Gazebo simulation and validated in different experiments using a prototype walking excavator machine. The proposed method revolutionizes operator control of these versatile but complex multi-purpose vehicles: instead of manual and coordinatively very demanding cylinder position adjustment, the operator can command simple high-level commands like cabin pose. Furthermore, it significantly reduces peak forces in the cylinders and at the contact points, which causes less damage to the mechanics and the ground.
\end{abstract}

\section{INTRODUCTION}

The ability to overcome highly uneven ground has put forth a variety of legged robots. In the construction sector, the advantage in mobility led to the development of walking excavators [1]. These multi-purpose machines are deployed in all kinds of special scenarios that require advanced mobility such as in mountains, in river beds, or other hardly accessible areas. The object of research in this projects is a Menzi Muck M545 (Fig. 1), a twelve ton heavy four legged machine with three degrees of freedom per leg and actuated wheels at the end-effector.

Today, the operation of walking excavators is still very similar to machines like the GE Walking Truck developed in the 1960's [2]. For all locomotion maneuvers, the human operators must manually synchronize the motion of the four legs, meaning that they have to manually control the position of each of the three cylinders per leg by opening and closing a hydraulic valve. This challenging work requires very skilled operators, particularly when moving forward and excavating at the same time. The training of new operators takes a long time, which limits the utility of these vehicles. Moreover, the

1 M. Hutter, P. Leemann, G. Hottiger are with the Robotic Systems Lab, ETH Zurich, Switzerland (mahutter@ethz.ch), ${ }^{2}$ R. Figi, S. Tagmann are with Menzi Muck AG, Switzerland (rfi@menzimuck.com), ${ }^{3}$ G. Rey, G. Small are with Moog inc., US (grey@moog.com)

Manuscript submitted October 14, 2015, resubmitted February 26, 2016, final revision June 3, 2016, final submission September 21, 2016. This research was supported by the Commission for Technology and Innovation (CTI) Switzerland through project 16004-1.

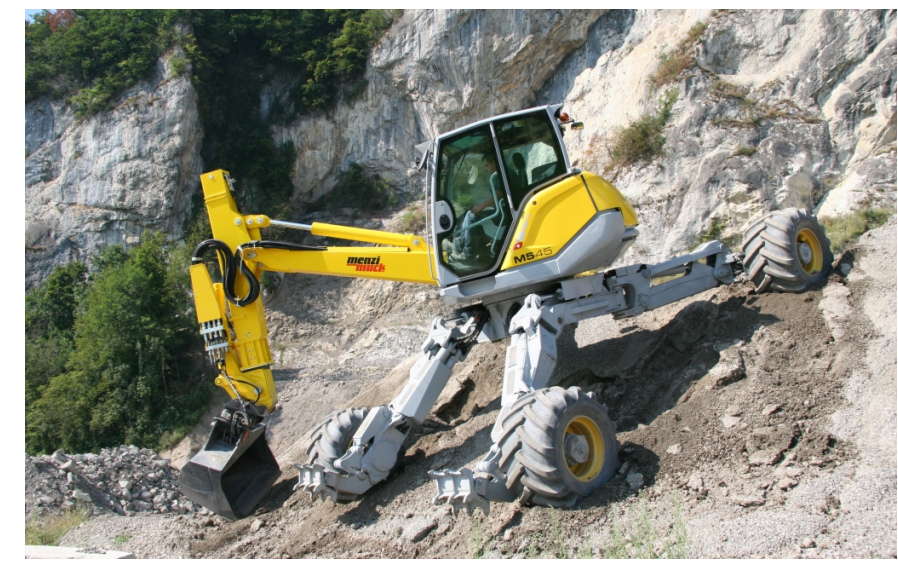

Fig. 1: Walking excavators are deployed for work in challenging environments.

permanent coordination necessary to drive the machine over bumpy terrain can be very exhausting.

The problem of placing a rigid, position controlled system on four legs is kinematically undefined. As a result, the entire body weight is supported by only three legs and already infinitesimally small changes in positions will lead to change in the contact situation. For machines like the walking excavator, which has the center of gravity ( $\mathrm{CoG}$ ) close to the geometric center of the contact points, almost the full load is taken by one diagonal axis. This implies high peak forces which can damage the terrain or wear off the suspension system of the excavator. Only the occurrence of compliance in the structure, at the contact points or in the actuation ensures that all legs are supporting the machine. This is often realized with a passive suspension system of individual wheels (c.f. car suspension) or by a kinematic coupling (e.g. [3]), which can significantly improve climbing capabilities. In hydraulics, the latter method is often realized by a so-called swing axle at the front or the rear suspension [1]. Unfortunately, all these passive suspension systems limit the versatility of the machines.

The goal of the presented work is to implement an active contact force adaptation on a walking excavator to overcome the limitations of position controlled machines and to path the road for autonomous operation respectively locomotion of such vehicles over rough terrain. To this end, we transfer force control technologies as they are used today by the most advanced multi-legged robots such as Big Dog [4] (and its successors), HyQ [5], Cheetah [6], StarlETH [7] or bipedal systems like Atlas [8] or Valkyrie [9]. All these systems actively regulate their (dynamic) behavior by precisely modulating the ground reaction forces.

In a first step, we develop the necessary tools to accurately regulate the force of a hydraulic cylinder. In contrast to 
high-performance robotic systems like Sarcos humanoid [10] or HyQ quadruped [5], which build their force control on additional joint force/torque sensors [11], we implement a hydraulic pressure control with a simple Stribeck friction compensation [12]. Based on one a high performant industrial valves, we created in intelligent control module with integrated electronics for fast pressure feedback control. The proposed setup exhibits very fast cylinder force tracking $(100 \mathrm{~Hz})$ with high accuracy. In contrast to similar robots like COMET-VI [13], [14], this allows us to directly regulate the joint force without underlying cylinder position feedback loop. Despite large model uncertainty, we are able to track the ground contact force with an accuracy of about 3\% of the machine weight. In a second step, we design a control algorithm that optimally distributes the contact force as a function of the robot state, namely configuration of the legs and position and orientation of the base. In view of the relatively slow motion of the walking excavator, we simplify the robot as quasistatic system and implement a virtual model approach [15], i.e. virtual forces in combination with Jacobian transposed method [16]. Methods based on this principle of finding contact forces that apply certain reaction on the body have been often applied by many researchers for balancing tasks of humanoid robots [15], [17], [18], or multi-legged systems [16], [19], and has proven to be a very capable method even when applied to highly dynamic locomotion [20], [21]. In case of redundancy of the contact situation, it is possible to state the force and moment equilibrium of the complete system as weighted optimization problem (e.g.[18], [17], [22]) or as prioritized formulation as commonly used by the whole body control community (e.g. [23], [24], [25], [26]). In comparison to these methods that often rely on fully force/torque controllable robotic systems, we exploit the kinematic structure of the legs of our system as well as the horizontal alignment to further simplify the problem and to reduce the set of required actuators. In the present work, we control only the legs, i.e. the chassis of the robotic system. The configuration of the significantly heavier upper machine (UM), i.e. the cabin, arm and bucket, is not known and considered as disturbance, which is approximated as accurately as possible for the quasi-static case. The proposed method is tested in a Gazebo simulation and validated on a full-scale M545 excavator. Thereby we are able to accurately track ground reaction forces and to precisely adjust the cabin pose while driving on uneven terrain and with significant disturbances coming from a moving $\mathrm{CoG}$ at the upper machine.

\section{Force Control of A Hydraulic Cylinder}

Key element to an optimal ground contact force distribution is precise cylinder force control [27]. However, in contrast to some of the best hydraulic walking robots (e.g. [5]), economic reasons and system robustness prevent the use of additional force sensors (Fig. 2). In fact, force control must be achieved based on pressure readings $F_{h}$ and hence requires to estimate the load force $F_{L}$ respectively friction force $F_{f}$ based on staterespectively piston velocity $\dot{x}_{p}$ feedback.

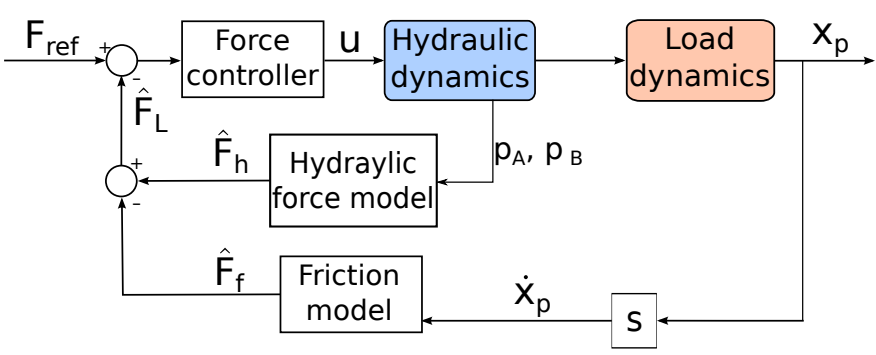

Fig. 2: Hydraulic force control: In the absence of force sensors, the output force $\left(F_{L}\right)$ must be estimated from the hydraulic force $\left(F_{h}\right)$.

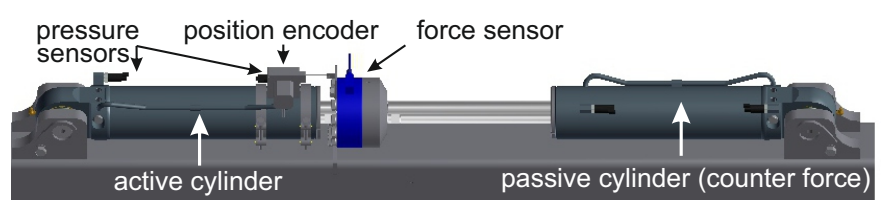

Fig. 3: A single axis test setup equipped with a precise load cell was used to characterize the performance and to tune the controller [28].

\section{A. Test Bench Setup}

To characterize the force control behavior and to tune controllers, we developed a single axis test bench (Fig. 3) consisting of an active cylinder with pressure sensors (Rexroth RE 95138), a load cell to measure the output force (MecSense PC4), a passive cylinder to produce the counter force, an incremental position sensor (Micro-Epsilon WPS-2100MK77) to measure the piston position and correspondingly its velocity and hydraulic flow, an external mobile pump of $4 \mathrm{~kW}$ electric power with constant supply pressure of 200 bars, which corresponds to an average pressure level we expect in the excavator.

\section{B. Friction Identification and Modeling}

To accurately control the mechanical force $\left(F_{L}\right)$ based on pressure readings in the chambers, a good friction model is required. Interestingly, the large scale cylinders employed in the M545 $\left(A=7.85 \cdot 10^{-3} \mathrm{~m}^{2}, F_{\max }=200 \mathrm{kN}\right)$ show very small relative friction with very limited stiction effects that contribute to less than $1 \%$ of the maximal force Fig. 4 The corresponding characteristics can be accurately captured

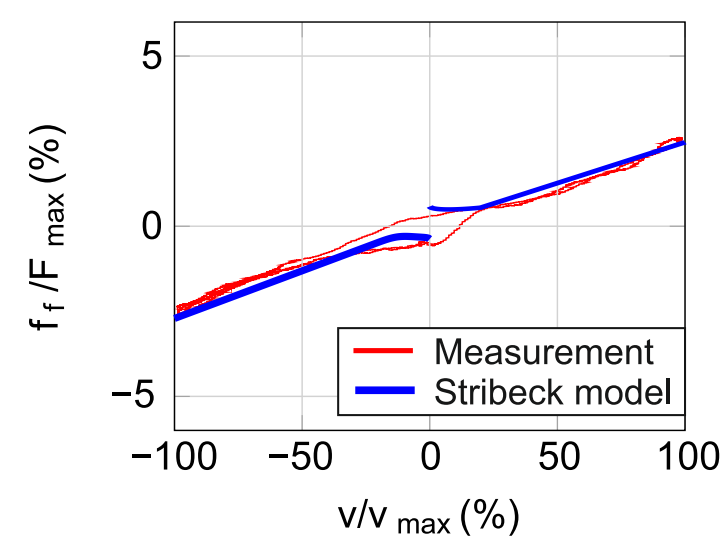

Fig. 4: The friction on the large scale cylinder can be accurately captured with a Stribeck model. 

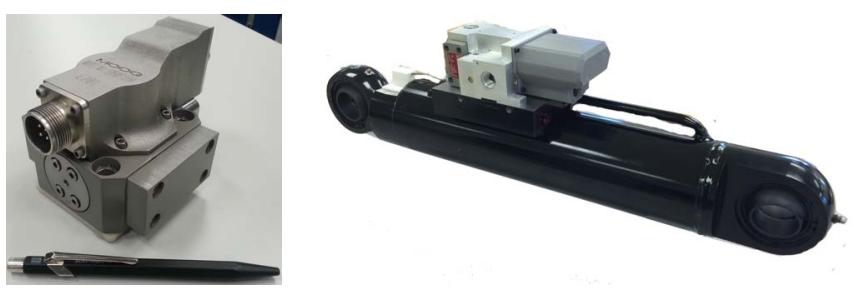

(a) The valve with in- (b) ... integrated on the cylinder, with a piston cluded electronics ... position encoder.

Fig. 5: Integrated control module (ICM)

by a simple Stribeck curve [12]. The frictional effects seem significantly less important than what has been reported in other studies like [29], [30]. In contrast to other solutions implemented in excavators [31], accurate force control seems possible without an additional load cell as also shown in [32].

\section{Integrated Control Module}

Standard valves of construction site machines often have a closed center spool with large overlaps (often in the range of about 50\%). This is an easy and inexpensive way to minimize the flow through the valve when it is closed and thus ensuring that the cylinder does not move. However, when controlling the valve in pressure mode and hence around zero flow, this overlap can be compensated but has to be crossed each time the measured signal crosses its reference. Beside energetic inefficiency, the overlap leads to a deadband, which significantly decreases the controller performance or requires complex setups with a dual valve configuration as proposed in [28].

To overcome these problems, we developed a highly integrated control module (ICM) based on a Moog G761 servo valve, one of the fastest hydraulic valves of its size. Similarly to the servo valves used in robots like HyQ [5] or Boston Dynamics Big Dog [4], this precisely manufactured valve features zero overlap. The module is enhanced with pressure transducers and an integrated STM32F407 microprocessor running the low level force control loop at a frequency of $10 \mathrm{kHz}$. To ensure fast system reaction and to overcome some of the safety issues of broken hoses, the module is directly mounted on the cylinder.

\section{Performance Evaluation}

We compared the performance of a standard proportional valve with an overlapped spool (Duplomatic BLS6) and the prototype ICM version. For pressure regulation, we implemented a simple PI controller:

$$
u_{v}=P I(\Delta p) .
$$

From the step responses (Fig. 6), the bandwidth of the standard valve is estimated about $5 \mathrm{~Hz}$ and about $100 \mathrm{~Hz}$ for the ICM. For this comparison, only the hydraulic force control loop was considered and the piston of the cylinder was blocked. The influence of the friction compensation, as outlined in Sec. [II-B, with the ICM connected to a cylinder

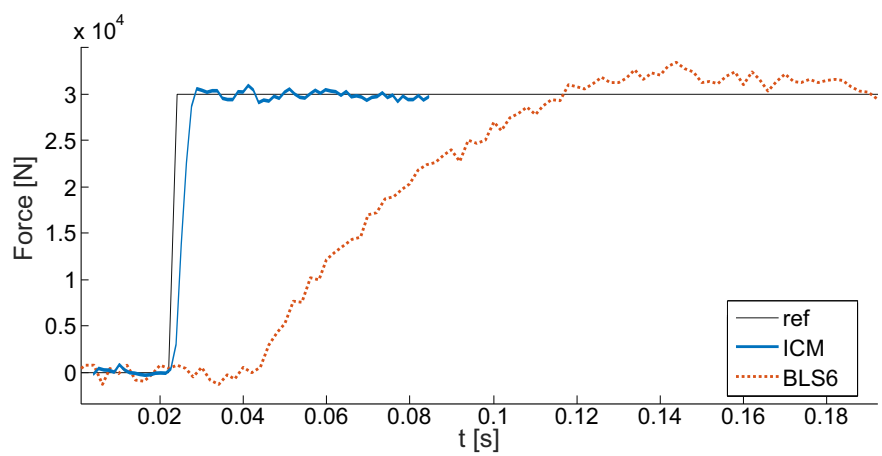

Fig. 6: Scaled step response comparison between the BLS6 Duplomatic valve with the ICM on a fixed displacement setup.

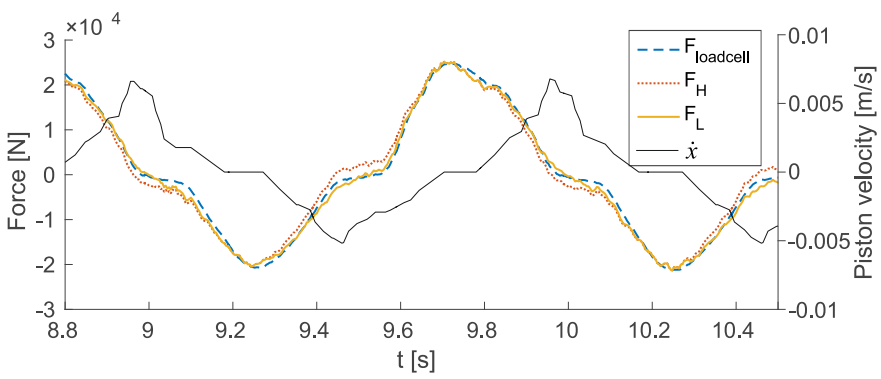

Fig. 7: Comparison of the load force measured with a loadcell $\left(F_{\text {load }}\right)$ and estimated with $\left(F_{L}\right)$ and without $\left(F_{H}\right)$ friction compensation.

whose piston is allowed to carry out small movements is depicted in Fig. 7. The highest piston velocity and thus largest influence of the friction compensation can be observed during the zero-crossings of the applied force.

\section{EXCAVATOR MODEL}

The model of the excavator (Fig. 1) is split in two components, namely the chassis and the upper machine (UM) consisting of the cabin, arm and bucket. Only the chassis is equipped with sensors and subject to be controlled, while the influence of the UM is considered as large, non-modeled error applying substantial disturbance forces and moments to the chassis. The chassis can be modeled as a floating-base multi-body system with $n_{q}$-dimensional generalized coordinate vector:

$$
\mathbf{q}=\left(\begin{array}{l}
\mathbf{q}_{b} \\
\mathbf{q}_{r} \\
\mathbf{q}_{j}
\end{array}\right),
$$

which consists of the $n_{b}=3$-dimensional vector $\mathbf{q}_{b}$ describing the unactuated base position $\in \mathbb{R}^{3}$, the $n_{r}$-dimensional vector $\mathbf{q}_{r}$ describing the base orientation $\in S O(3)$, and of the $n_{j}$ dimensional vector $\mathbf{q}_{j}$, representing the joint coordinates of the chassis $\left(n_{j}=12\right)$.

The kinematics of the chassis can be described by the state vector $\mathbf{q}$ and a set of link lengths $\mathfrak{L}$. With these parameters, a point $P$ of the system with its origin at point $B$ can be generally expressed as

$$
{ }_{B} \mathbf{r}_{I P}={ }_{B} \mathbf{r}_{I P}(\mathbf{q}, \mathfrak{L})=\mathbf{R}_{B I}\left(\mathbf{q}_{r}\right){ }_{I} \mathbf{r}_{I B}\left(\mathbf{q}_{b}\right)+{ }_{B} \mathbf{r}_{B P}\left(\mathbf{q}_{j}, \mathfrak{L}\right),
$$


whereby $I$ and $B$ represent the inertial and body fixed frames, respectively, and $\mathbf{R}_{B I}$ the rotation matrix from $I$ to $B$. Using basic kinematic rules, the velocity at point $P$ results to

$$
{ }_{B} \dot{\mathbf{r}}_{I P}=\mathbf{R}_{B I} \dot{\mathbf{q}}_{b}+{ }_{B} \dot{\mathbf{r}}_{B P}\left(\mathbf{q}_{j}\right)-{ }_{B} \tilde{\mathbf{r}}_{B P B} \boldsymbol{\omega}_{I B},
$$

with ${ }_{B} \tilde{\mathbf{r}}_{B P}$ being the cross multiplication matrix ${ }_{B} \tilde{\mathbf{r}}_{B P B} \boldsymbol{\omega}_{I B}=-{ }_{B} \boldsymbol{\omega}_{I B} \times{ }_{B} \mathbf{r}_{B P}$. By using the rotation parametrization depending mapping matrix $\mathbf{E}$ from generalized rotational velocities $\dot{\mathbf{q}}_{r}$ to angular velocities of the base $\left({ }_{B} \boldsymbol{\omega}_{I B}=\mathbf{E}\left(\mathbf{q}_{r}\right) \dot{\mathbf{q}}_{r}\right)$, and the relative Jacobian $\mathbf{J}_{B P, j}\left(\mathbf{q}_{j}\right)=\frac{\partial \mathbf{r}_{B P}\left(\mathbf{q}_{j}\right)}{\partial \mathbf{q}_{j}}$, the Jacobian ${ }_{B} \mathbf{J}_{I P}$ is

$$
{ }_{B} \mathbf{J}_{I P}=\left[\mathbf{R}_{B I},-{ }_{B} \tilde{\mathbf{r}}_{B P} \mathbf{E}\left(\mathbf{q}_{r}\right),{ }_{B} \mathbf{J}_{B P}\left(\mathbf{q}_{j}\right)\right] .
$$

The equation of motion of the system describing its dynamics can be written as

$$
\mathbf{M} \ddot{\mathbf{q}}+\mathbf{b}+\mathbf{g}+\boldsymbol{\lambda}_{c}=\mathbf{S}^{T} \boldsymbol{\tau}+\boldsymbol{\lambda}^{d i s t},
$$

with the mass matrix $\mathbf{M}(\mathbf{q})$, the Coriolis and centrifugal vector $\mathbf{b}(\mathbf{q}, \dot{\mathbf{q}})$, the gravitational vector $\mathbf{g}(\mathbf{q})$, the actuator torques $\boldsymbol{\tau}$ and the selection matrix $\mathbf{S}=\left[\mathbf{0}_{n_{j} \times\left(n_{b}+n_{r}\right)}, \mathbf{I}_{n_{j} \times n_{j}}\right]$ separating the actuated joint coordinates $\mathbf{q}_{j}$ and unactuated base coordinates $\mathbf{q}_{b}$ and $\mathbf{q}_{r}$. The generalized contact force vector $\boldsymbol{\lambda}_{c}$ is given by

$$
\boldsymbol{\lambda}_{c}=\sum_{i=1}^{n_{c}} \mathbf{J}_{I C_{i}}^{T} \mathbf{F}_{C_{i}}
$$

with the contact forces $\mathbf{F}_{C_{i}}$ at the $n_{c}$ contact points. The gravitational part $\mathbf{g}$ is given by

$$
\mathbf{g}=-\sum_{i=1}^{n} \mathbf{J}_{I S_{i}}^{T} \mathbf{F}_{g_{i}}
$$

with the Jacobian $\mathbf{J}_{I S_{i}}$ evaluated at the center of gravity $S$ of every link $i \in n$. All forces caused by the motion and configuration of the cabin and arm are lumped in the generalized disturbance force vector $\lambda^{d i s t}$. Due to the kinematic tree structure, there are no direct coupling effects from the cabin and arm to the legs but only through the base. Hence, the disturbance force is given by:

$$
\boldsymbol{\lambda}^{\text {dist }}=\left[\begin{array}{c}
\lambda_{b}^{d i s t} \\
\lambda_{r}^{\text {dist }} \\
\mathbf{0}
\end{array}\right]
$$

\section{Force Distribution And Orientation Control}

The motion of the torque controllable excavator is regulated by changing the ground contact force and hence the net force and moment acting on the systems base.

\section{A. Quasi-static Simplification}

Since the large and heavy excavator machine and in particular its chassis is moving relatively slow, the system dynamics can be approximated quasi-static and hence the controller does not have to compensate for acceleration (M̈̈), Coriolis and centrifugal (b) parts. This quasi-static assumption reduces (6) to

$$
\sum_{i=1}^{n_{c}} \mathbf{J}_{I C_{i}}^{T} \mathbf{F}_{C_{i}}-\sum_{i=1}^{n} \mathbf{J}_{S_{i}}^{T} \mathbf{F}_{g_{i}}=\mathbf{S}^{T} \boldsymbol{\tau}+\boldsymbol{\lambda}^{d i s t} .
$$

By using the Jacobian separation from (5) and the disturbance vector from (9), we can extract

$$
\begin{aligned}
\sum_{i=1}^{n_{c}} \mathbf{R}_{I B B} \mathbf{F}_{C_{i}}-\sum_{i=1}^{n} \mathbf{R}_{I B B} \mathbf{F}_{g_{i}} & =\boldsymbol{\lambda}_{b}^{d i s t} \\
\mathbf{E}\left(\sum_{i=1}^{n_{c}}{ }_{B} \tilde{\mathbf{r}}_{B C_{i} B} \mathbf{F}_{C_{i}}-\sum_{i=1}^{n}{ }_{B} \tilde{\mathbf{r}}_{B S_{i} B} \mathbf{F}_{g_{i}}\right) & =\boldsymbol{\lambda}_{r}^{d i s t} \\
\sum_{i=1}^{n_{c}}{ }_{B} \mathbf{J}_{I C_{i} B}^{T} \mathbf{F}_{C_{i}}-\sum_{i=1}^{n}{ }_{B} \mathbf{J}_{B S_{i} B}^{T} \mathbf{F}_{g_{i}} & =\boldsymbol{\tau} .
\end{aligned}
$$

The first two equations represent the force (11) and torque (12) equilibrium evaluated for the full system, whereby the disturbance forces from the UM are unknown.

\section{B. Small Roll/Pitch Simplification}

For relatively small roll/pitch angles (the chassis is mostly kept level) and since most of contact force is caused by supporting the machine $\left({ }_{I} F_{z}>>{ }_{I} F_{x},{ }_{I} F_{y}\right)$, the following simplifications can be further applied:

$$
\begin{aligned}
{ }_{B} \mathbf{F}=\mathbf{R}_{B I I} \mathbf{F} & \approx{ }_{I} \mathbf{F} \\
\mathbf{J}^{T} \mathbf{F} & \approx \mathbf{j}_{z}^{T} F_{z}=\left(\frac{\partial r_{z}}{\partial \mathbf{q}}\right)^{T} F_{z},
\end{aligned}
$$

such that (11)-(13) simplifies to:

$$
\begin{array}{r}
\sum_{i=1}^{n_{c}} F_{(C z)_{i}}-\sum_{i=1}^{n} m_{i} g=\lambda_{z}^{d i s t} \\
\sum_{i=1}^{n_{c}}\left(\begin{array}{c}
B \\
{ }_{B} y_{B C_{i}} \\
-{ }_{B} x_{B C_{i}}
\end{array}\right) F_{(C, z)_{i}}-\sum_{i=1}^{n}\left(\begin{array}{c}
{ }_{B} y_{B S_{i}} \\
-{ }_{B} x_{B S_{i}}
\end{array}\right) m_{i} g=\lambda_{(x, y)}^{\text {dist }} \\
\sum_{i=1}^{n_{c}} \mathbf{j}_{(B C, z)_{i}}^{T} F_{(C z)_{i}}-\sum_{i=1}^{n}{ }_{B} \mathbf{j}_{(B S, z)_{i}}^{T} m_{i} g=\boldsymbol{\tau}
\end{array}
$$

This is a generic formulation of the static equilibrium for a leveled machine with low internal horizontal contact forces. In fact, what remains is the force equilibrium in vertical direction (16) and the torque equilibrium around two horizontal axis (17). If the cabin and arm are not or only slowly moved, the disturbance force in vertical direction $\lambda_{z}^{\text {dist }}$ can be approximated by the total weight of the UM $\lambda_{z}^{d i s t}=m_{U M} g$. The moment disturbances $\lambda_{(x, y)}^{\text {dist }}$ are caused by the relatively large CoG offset from the base center $( \pm 1.5 \mathrm{~m})$. Since orientation of the UM is completely unknown, there is no model-based approximation for $\boldsymbol{\lambda}_{(x, y)}^{\text {dist }}$ and these effects must be entirely compensated by the feedback controller.

\section{Kinematic Particularity of M545}

The kinematic structure of the front and hind legs of the M545 consists of two abduction/adduction degrees of freedom (rotation around vertical axis) and one flexion/extension degree of freedom (rotation around horizontal axis). Both legs feature a different arrangement and a parallelogram mechanism. In leveled situation, the vertical motion of the wheel is not depending on the two abduction/adduction degrees of freedom. 

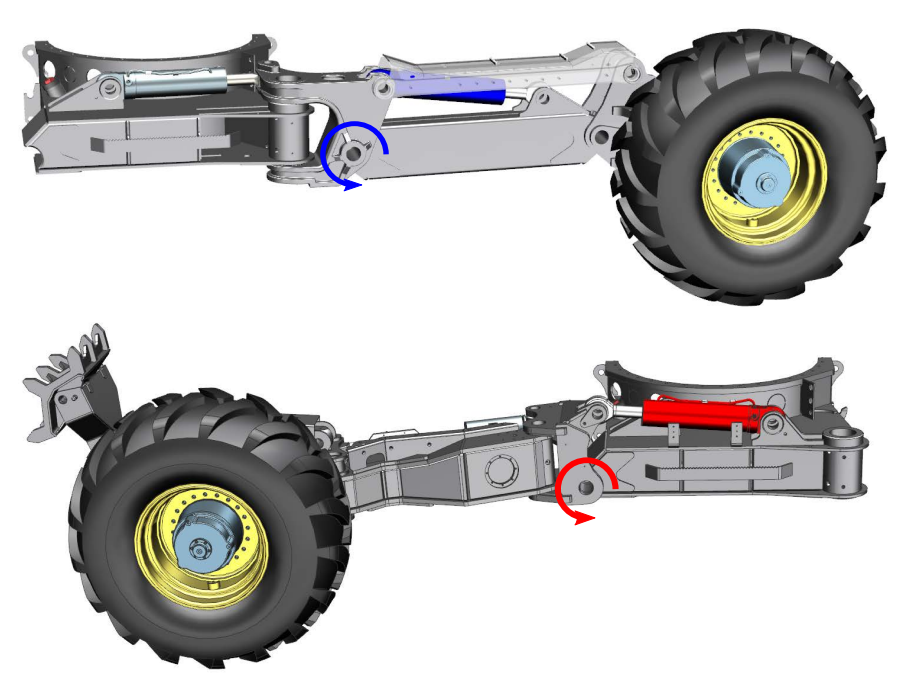

Fig. 8: Front (top) and hind leg (bottom) with colored flexion joints and cylinders.

Therefore, for many maneuvers it is sufficient to automate the adjustment the four flexion/extension joints of all legs (see colored cylinders in Fig. 8)

\section{Virtual Model Control with Force Optimization}

A classical quasi-static control approach is virtual model control [15]. Thereby, virtual forces and moments

$$
\begin{aligned}
{ }_{B} F_{v} & =P I D^{p}\left({ }_{B} z^{\text {des }}-{ }_{B} z_{b}\right) \\
{ }_{B} \mathbf{T}_{v} & =P I D^{r}\left({ }_{B} \boldsymbol{\varphi}^{\text {des }}-{ }_{B} \boldsymbol{\varphi}\right)
\end{aligned}
$$

as a function of proportional, integral, and derivative gains are applied to the main body to push or pull the system to the desired height and orientation. Together with the previously derived static equilibrium (16) and (17), this results in the following equation to determine the necessary ground reaction forces:

$$
\begin{array}{r}
\underbrace{\left[\begin{array}{ccc}
1 & \ldots & 1 \\
{ }_{B} y_{B C_{1}} & \ldots & { }_{B} y_{B C_{4}} \\
-{ }_{B} x_{B C_{1}} & \ldots & -{ }_{B} x_{B C_{4}}
\end{array}\right]}_{\mathbf{A}} \underbrace{\left(\begin{array}{c}
F_{(C z)_{1}} \\
F_{(C z)_{2}} \\
F_{(C z)_{3}} \\
F_{(C z)_{4}}
\end{array}\right)}_{\mathbf{b}}, \underbrace{\sum_{i=1}^{n} m_{i} g\left(\begin{array}{c}
1 \\
{ }_{B} y_{B S_{i}} \\
-{ }_{B} x_{B S_{i}}
\end{array}\right)+\left(\begin{array}{c}
{ }_{B} F_{v} \\
{ }_{B} \mathbf{T}_{v}
\end{array}\right)+\left(\begin{array}{c}
m_{U M} g \\
0 \\
0
\end{array}\right)},
\end{array}
$$

whereby the last vector of $\mathbf{b}$ corresponds to the approximated gravity compensation of the UM.

With four legs in ground contact, the A-matrix features a 1-dimensional null-space $\mathcal{N}(A)$ that allows to change the contact force distribution without changing the reaction on the main body. This allows to additionally optimize contact force objectives or to comply with saturation effects by formulating

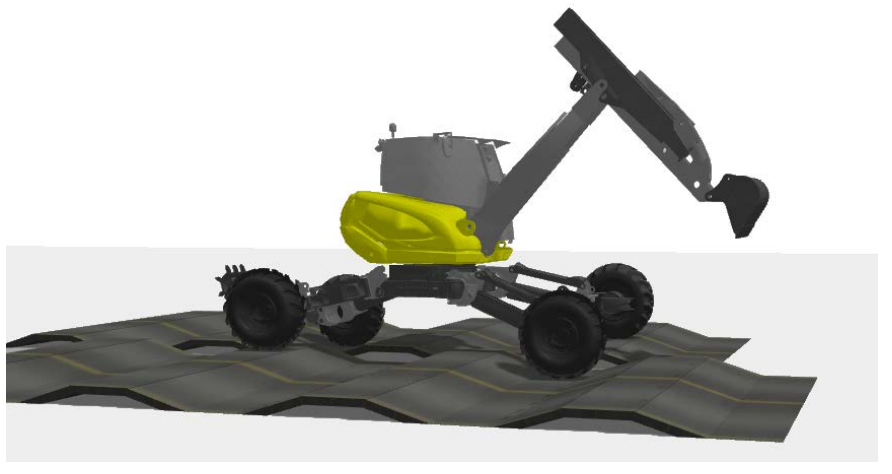

Fig. 9: Simulation test environment with a bumpy terrain.

a prioritized quadratic optimization problem with equality and in-equality constraints like e.g.:

$$
\begin{array}{rll}
\min & \mathbf{x}^{T} \mathbf{W} \mathbf{x} & \\
\text { s.t. } & F_{(C z)_{i}}>F_{c, \min } & \text { (Prio 1) } \\
& F_{(C z)_{i}}<F_{c, \max } & \text { (Prio 1) } \\
& \mathbf{A x}-\mathbf{b}=\mathbf{0} & \text { (Prio 2) }
\end{array}
$$

The solution of this optimization problem yields the minimal contact forces (weighted with the matrix $\mathbf{W}$ ) that achieve the desired virtual forces acting on the system under the constraints that the contact forces remain within certain limits. Due to the prioritization, the virtual forces (equality constraint with Prio 2) are only reached in a least square optimal manner in case of force saturation. The resulting optimal vertical ground contact forces can be subsequently transferred to joint torques respectively cylinder forces using the Jacobian transposed method from (18). The same equation can also be used to extend 22 with constraints or optimization objectives for cylinder forces respectively joint torques.

\section{Simulations}

The above described control methods are implemented and tested using the Gazebo simulation environment together with the ROS libraries.

The performance of the controllers is evaluated in a continuous bumpy terrain experiment (Fig. 9). The bumps have a height of $0.25 \mathrm{~m}$ and an interval of $3.4 \mathrm{~m}$. Thereby, we imply the control objective of keeping the chassis at the desired attitude of zero pitch and zero roll. Without adapting the leg suspension to the ground, the chassis largely deviates in pitch and roll angles (Fig. 10, yellow). Moreover, the load is almost entirely supported by the diagonal axis. When controlling the hind legs as proposed in [28], but keeping the front legs manually operated, the chassis pitch angle can be automatically adjusted while the roll angle remains controlled by the operator (red). The virtual model controller with four automated legs is able to completely compensate for the bumpy terrain and keeps the chassis leveled (blue).

\section{EXPERIMENTS}

To test the proposed chassis balancing method, we conducted a series of experiments on a real excavator. The experiments were chosen to resemble some of the most relevant 


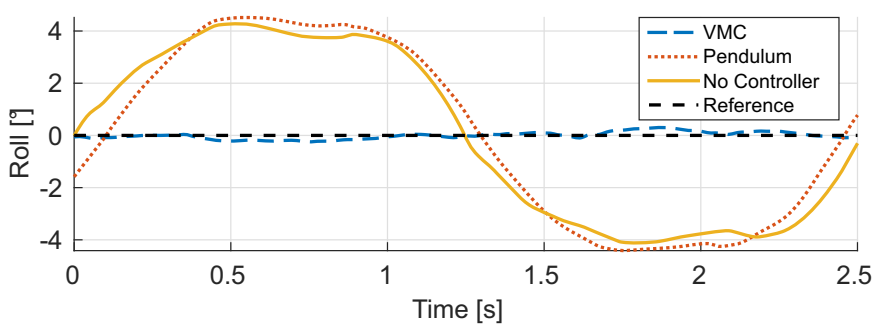

(a) Chassis roll

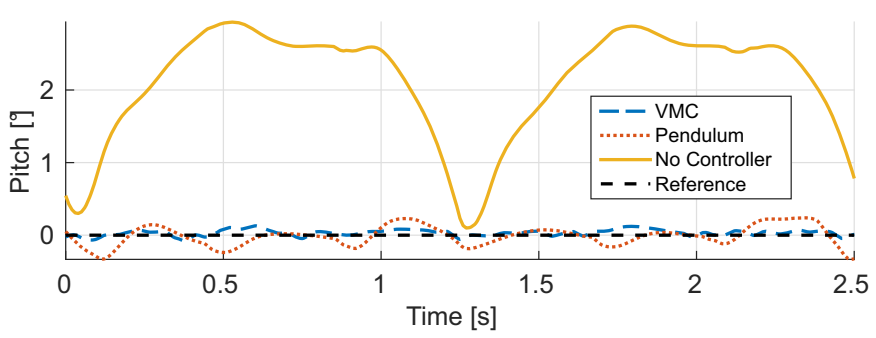

(b) Chassis pitch

Fig. 10: Driving over a bump with fixed legs (yellow) has a significant influence on the chassis roll and pitch angle. By controlling the two hind flexion cylinders (red, [28]) the chassis pitch can be stabilized. Using a virtual model controller on all four flexion cylinders (blue), the chassis can be leveled completely, without any input from the operator.

working task in daily operation. The results in chassis pose control and ground contact force distribution are compared to the simplified setup presented in [28] as well as to manual control by a trained operator. In our setup, an IMU with integrated Kalman filter is used to estimate the chassis orientation (roll and pitch). Potentiometers measure the angles of all chassis joints and are used to estimate ground height. The joint angles and orientation of the UM are not measured and therefore the $\mathrm{CoG}$ of the excavator is unknown. The system model including individual segment masses and CoG locations were directly taken from $\mathrm{CAD}$ and the machine manual without further identification.

\section{A. Force Distribution Evaluation}

1) Contact force measurements: To evaluate the accuracy of our model (18), the estimated ground contact forces from cylinder forces $F_{(c, z)_{i}}^{e s t}$ where compared to actual measurements provided by industrial balances placed underneath all wheels. Table $[\mathrm{I}$ shows the result from one of the two diagonal axis, whereby the UM is oriented to all four cardinal directions. Despite large model uncertainties (e.g. masses of the cylinders, hoses, oil etc. are ignored), unobservable internal (horizontal) forces, and low-accuracy joint position measurements from analog potentiometers, the force differences are less than $3 \%$ of the total body mass.

2) Cabin Pose Stability for Different Arm Directions: Since the unobservable rotation of the UM significantly shifts the overall CoG location, the chassis must permanently adapt the ground reaction force distribution to keep horizontal level. In this experiment, the $\mathrm{UM}$ was moved from the right front $\left(30^{\circ}\right)$ to the left front $\left(-30^{\circ}\right)$ and back again. By adapting the force

\begin{tabular}{|c|c|c|c|c|}
\hline & $F_{R F}^{\text {balance }}$ & $F_{R F, z}^{\text {est }}$ & $F_{L H}^{\text {balance }}$ & $F_{L H, z}^{\text {est }}$ \\
\hline Front & $28050 \mathrm{~N}$ & $27050 \mathrm{~N}$ & $25500 \mathrm{~N}$ & $23600 \mathrm{~N}$ \\
\hline Right & $29920 \mathrm{~N}$ & $28700 \mathrm{~N}$ & $25600 \mathrm{~N}$ & $22600 \mathrm{~N}$ \\
\hline Hind & $17070 \mathrm{~N}$ & $14900 \mathrm{~N}$ & $36980 \mathrm{~N}$ & $37800 \mathrm{~N}$ \\
\hline Left & $14810 \mathrm{~N}$ & $13560 \mathrm{~N}$ & $34920 \mathrm{~N}$ & $37140 \mathrm{~N}$ \\
\hline
\end{tabular}

TABLE I: Measured (industrial balance) and estimated (cylinder pressure) force on two legs for four different cardinal directions of the UM.

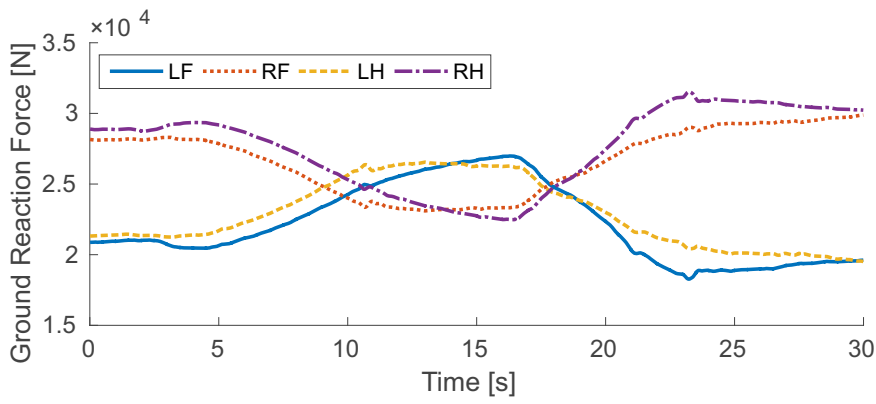

(a) Distributed forces

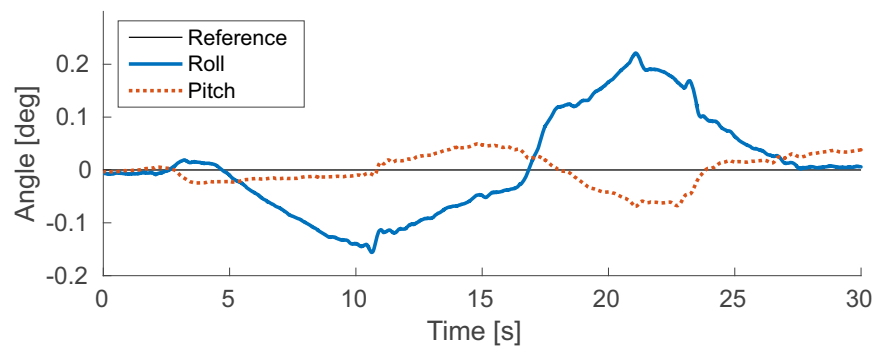

(b) Chassis orientation

Fig. 11: When shifting the CoG of the excavator by moving the UM, the contact force distribution is adapted (a), while the chassis attitude errors are kept very small (b).

distribution (Fig. 11(a), the orientation of the chassis attitude can be kept at less than $0.2^{\circ}$ from the leveled configuration (Fig. 11(b)).

3) Contact Force Limitation: The same experiment was repeated including a constraint that artificially limits the maximal contact force of the left front (LF) leg to $25 \mathrm{kN}$. As illustrated in Fig. 12(a), the force distribution looks similar in the areas without limitation. As soon as the limit of the LF leg is reached, the other legs (in particular the other diagonal axis) take more load such that the cabin remains leveled (Fig. 12(b)].

\section{B. Chassis Height and Orientation Tracking}

Changing level of the machine requires to move all four flexion cylinders in the front and back. During manual control, the operator needs to open the corresponding valves using two thumb joysticks per hand. Hence, the two front and two rear joints are usually sequentially moved (Fig. 13(a), yellow), leading to tilting of the chassis (Fig. 13(b) yellow). With the method presented in [28], the hind legs automatically follow the commanded front motion such that the machine automatically keeps its pitch orientation (Fig. 13(b), red). The virtual model control method allows to directly command the 


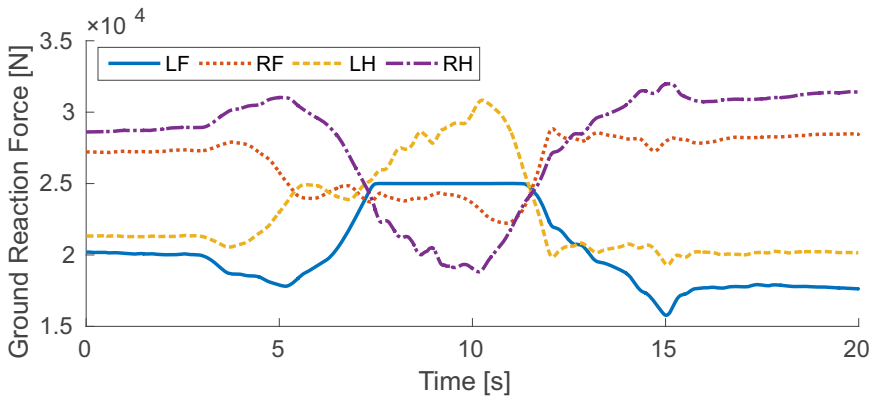

(a) Distributed forces

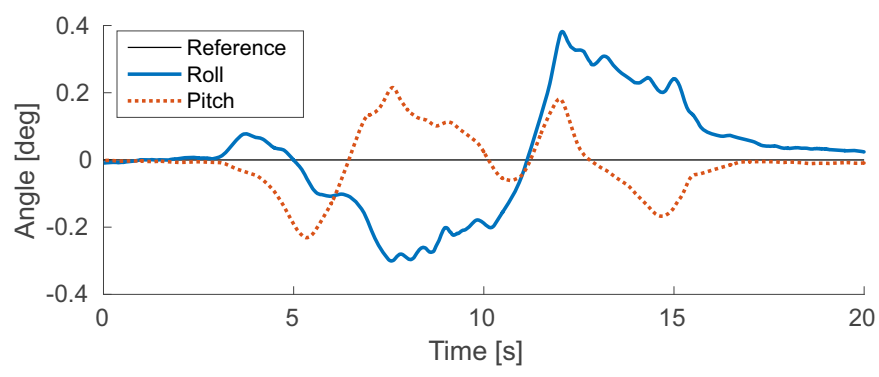

(b) Chassis orientation

Fig. 12: The same experiment as shown in Fig. 11 was performed while limiting the contact force of the left front leg to $25 \mathrm{kN}$. While When moving the UM to the left, the LF contact force enters saturation (a). Thus, most of the excavator weight is supported by the diagonal axis (LH-RF). Moreover, the chassis can be kept leveled during the whole experiment (b).

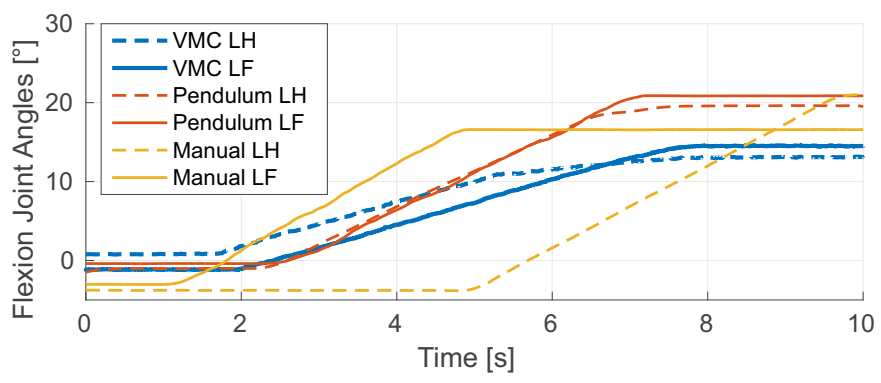

(a) Flextion joint angles of the left front and hind leg

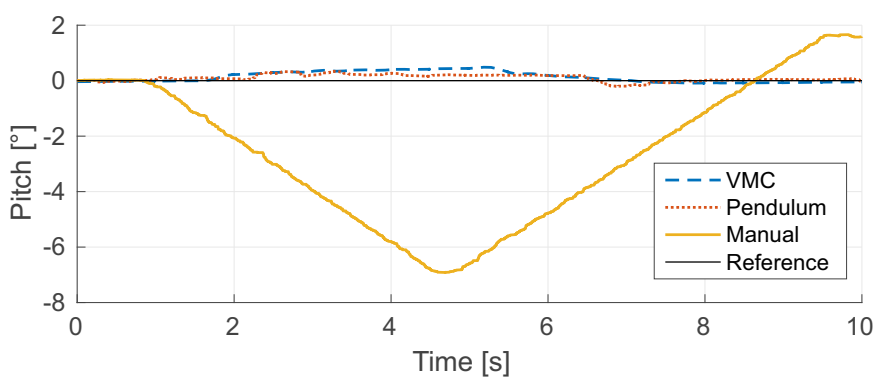

(b) Chassis pitch orientation

Fig. 13: Lifting the chassis with manual control (yellow) requires coordination of four thumb joysticks and can lead to large inclination errors (b) while the automated controllers keep the inclination almost constant with only two (red) respectively one (blue) user inputs.

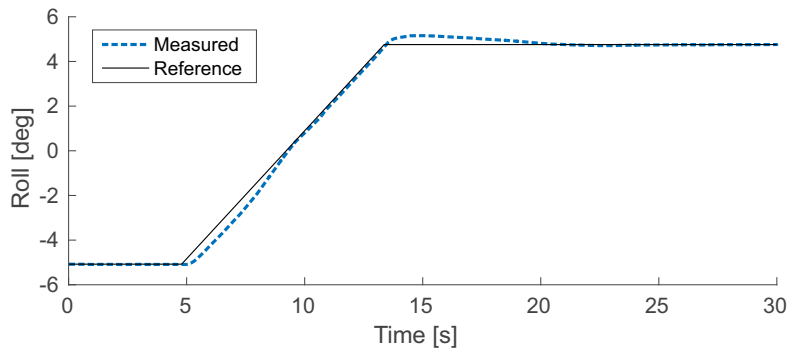

(a) Chassis roll

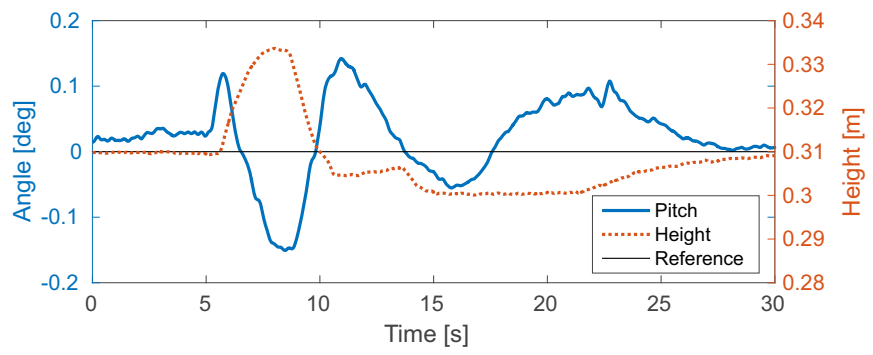

(b) Chassis pitch and height

Fig. 14: The chassis pitch and height (b) can be kept almost constant while applying a ramp reference signal (from -5 to +5 degrees) to the chassis roll angle (a). The overshoot is due to the integrators that are compensating for the unknown COG.

chassis height as a single input command that is accurately followed (Fig. 13, blue).

Furthermore, reference tracking was also evaluated for the body orientation. Roll angle experiment is depicted in Fig. 14 and the pitch response looks very similar. Due to the relatively large modeling error in CoG of the cabin and arm, and the correspondingly large integral values of in the virtual force, both experiments exhibit a small overshoot at the end of the reference tracking. The errors in chassis height and the remaining angle can be kept small in both experiments.

\section{Moving on Uneven Ground}

Finally, the excavator was tested when partially moving on an inclination (Fig. 15(a)). This is a typical working situation which involves adjustment of all four legs.

As illustrated in Fig. 15(b), even a very experienced driver is not capable of achieving an acceptable distribution of the weight on all four wheels when driving manually (yellow): The force distribution entirely shifts from the left to the right, meaning that the excavator is only supported by its diagonal axis.

As already demonstrated in [28], the supporting forces remain evenly distributed with activated balancing control (Fig. 15(b). Moreover, as depicted in Fig. 15(c) the required peak forces in the cylinders are significantly lower than in the manual control mode which clearly supports the claim of less damage to the mechanics and ground.

When comparing the human operator performance (yellow lines) and the pendulum axis experiments performed in [28] (red lines) with the control method proposed in this paper (blue lines), we see a clear improvement in maintaining a 


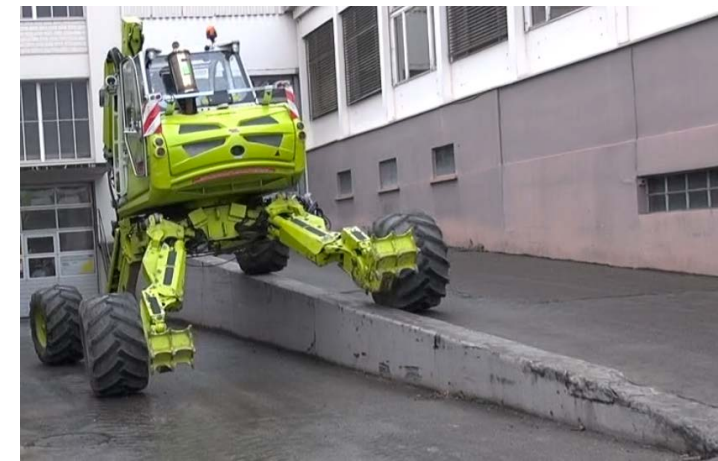

(a) Experimental setup for driving up and down a ramp.

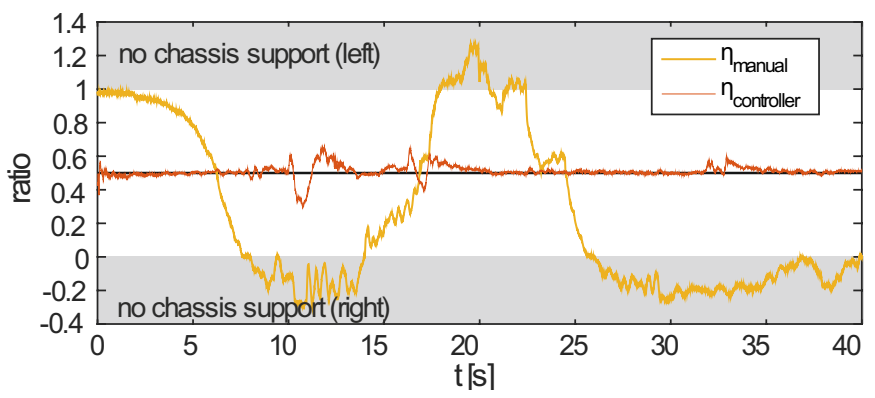

(b) Force distribution ratio of the hind cylinders

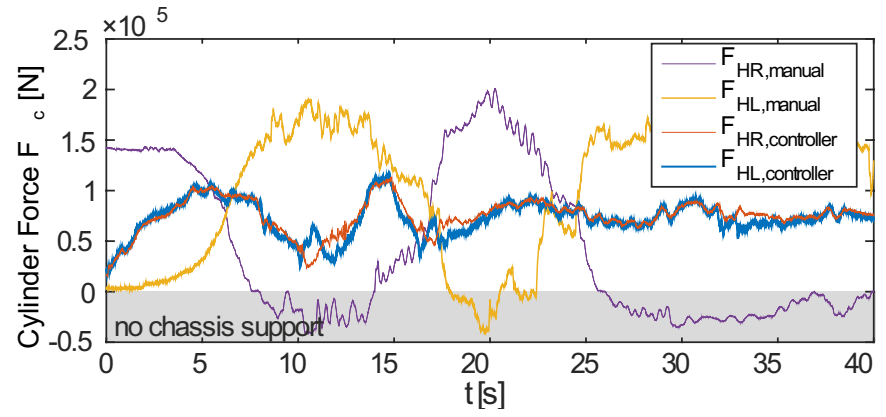

(c) Measured cylinder forces of the hind leg cylinders.

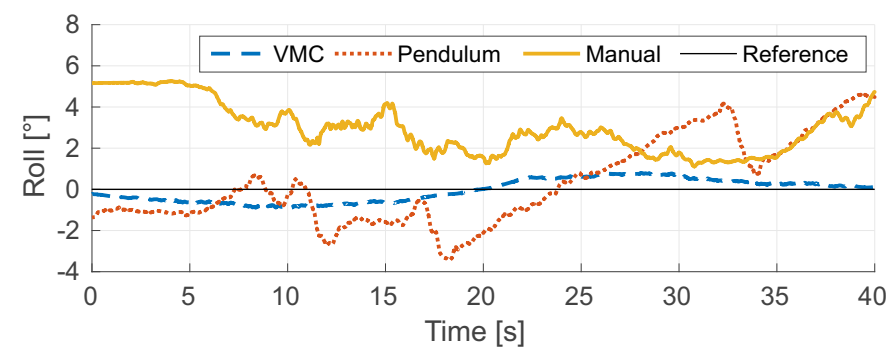

(d) Chassis roll orientation

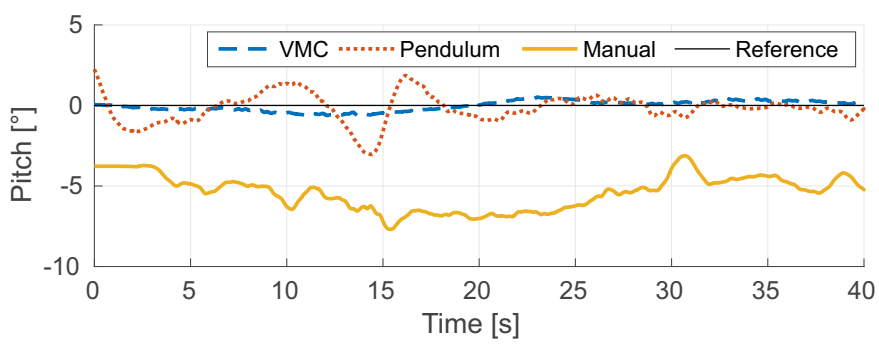

(e) Chassis pitch orientation

Fig. 15: Driving up and down the ramp. leveled chassis during the entire maneuver. Importantly, the proposed balancing strategy of this paper does not require any human input, such that the operator can fully concentrate on the working task.

\section{CONCLUSION}

A walking excavator is one of the most versatile construction machines. The versatility comes at a price: Controlling all joints of all legs while driving on uneven terrain requires skilled and specially trained operators. In this paper, we presented a force-based control method that (i) enables automatic adaptation of the legs to uneven terrain, (ii) an optimal force distribution among the legs, and (iii) simple high-level commands for body height and orientation. To this end, the authors developed a high-performance valve with integrated electronics for fast and precise pressure based cylinder force control. By approximating the system dynamics as quasi-static and by simplifying the force equilibrium around a level cabin orientation, the proposed virtual model control problem is simplified and finally formulated as prioritized contact force optimization with inequality constraints. While moving over uneven ground, it is possible to keep the chassis leveled or change its configuration using high-level task-space commands. The controller is able to robustly compensate for the large disturbances coming from the unknown UM configuration, which weighs more than $50 \%$ of the total machine weight and which can shift the overall $\mathrm{CoG}$ by more than $1.5 \mathrm{~m}$ in all directions. Having four legs in simultaneous ground contact, it is possible to exploit the contact redundancy and hence to optimally distribute the ground reaction forces. Extensive experiments with the, to the best our knowledge, world-wide first automated walking excavator chassis have not only demonstrated the applicability and the outstanding performance of the proposed method, but additionally confirmed the benefits with respect to lower peak forces on the mechanical structure as well as on the ground.

In future work, we plan to extend the proposed method in different ways. First of all, the influence of the motion and orientation of the cabin and arm can be better modeled by either instrumenting it or through online estimation from the measured cylinder forces. Second, we will evaluate the benefits when accounting for dynamic effects. Instead of the purely static approach used in the present work, the problem can be solved as a whole body control problem similar to our work with smaller legged robots [26]. To this end, the machine will be equipped with more accurate joint position sensors that enable better estimation of the complete machine state. Third, it is our goal to extend the high-performance cylinder force control technology from the four main cylinders of the chassis to all joints in order to develop the first fully force controllable walking excavator.

\section{ACKNOWLEDGEMENT}

The authors would like to thank Stefan Stevsic, Thiago Boaventura, Mark Hoepflinger, Markus Loher, and Christian Caduff for their contribution to this project. 


\section{REFERENCES}

[1] H. König, Maschinen im Baubetrieb. Springer Fachmedien Wiesbaden, 2014.

[2] P. G. Gonzales de Santos, E. Garcia, and J. Estremera, "Stability in Walking Robots," in Quadrupedal Locomotion. An Introduction to the Control of Four-legged Robots, vol. 1, pp. 33-54, Berlin: Springer, 2006.

[3] R. Y. Siegwart, P. Lamon, T. Estier, M. Lauria, and R. Piguet, "Innovative Design for Wheeled Locomotion in Rough Terrain," Robotics and Autonomous Systems, vol. 40, no. 2-3, pp. 151-162, 2002.

[4] M. Raibert, K. Blankespoor, G. Nelson, and R. Playter, "BigDog, the rough-terrain quadruped robot," in Proceedings of the 17th World Congress, pp. 10823-10825, 2008.

[5] C. Semini, N. G. Tsagarakis, E. Guglielmino, M. Focchi, F. Cannella, and D. G. Caldwell, "Design of HyQ - a hydraulically and electrically actuated quadruped robot," Proceedings of the Institution of Mechanical Engineers, Part I: Journal of Systems and Control Engineering, vol. 225, no. 6, pp. 831-849, 2011

[6] S. Seok, A. Wang, D. Otten, J. Lang, and S. Kim, "Design principles for highly efficient quadrupeds and implementation on the MIT Cheetah robot," in IEEE International Conference on Robotics and Automation (ICRA), pp. 3307-3312, 2013.

[7] M. Hutter, C. Gehring, M. A. Hopflinger, M. Blosch, and R. Siegwart, "Toward Combining Speed, Efficiency, Versatility, and Robustness in an Autonomous Quadruped," IEEE Transactions on Robotics, vol. 30, pp. 1427-1440, 2014.

[8] Boston "ATLAS Robot http://www.bostondynamics.com/robot_Atlas.html."

[9] N. A. Radford et al., "Valkyrie: NASA's First Bipedal Humanoid Robot," Journal of Field Robotics, vol. 32, pp. 397-419, 2015.

[10] G. Cheng, S.-h. H. Hyon, J. Morimoto, A. Ude, G. Colvin, W. Scroggin, S. Jacobsen, W. Way, and S. C. Jaconse, "CB: A Humanoid Research Platform for Exploring NeuroScience," in Humanoids, pp. 182-187, IEEE, 2006

[11] T. Boaventura, C. Semini, J. Buchli, M. Frigerio, M. Focchi, and D. G. Caldwell, "Dynamic torque control of a hydraulic quadruped robot," in IEEE International Conference on Robotics and Automation (ICRA), pp. 1889-1894, 2012.

[12] B. Armstrong-Helouvry, P. Dupont, and C. C. De Wit, "A survey of models, analysis tools and compensation methods for the control of machines with friction," Automatica, vol. 30, no. 7, pp. 1083-1138, 1994.

[13] A. Irawan and K. Nonami, "Optimal impedance control based on body inertia for a hydraulically driven hexapod robot walking on uneven and extremely soft terrain," Journal of Field Robotics, vol. 28, pp. 690-713, 2011.

[14] A. Irawan, K. Nonami, H. Ohroku, Y. Akutsu, and S. Imamura, "Adaptive Impedance Control with Compliant Body Balance for Hydraulically Driven Hexapod Robot," Journal of System Design and Dynamics, vol. 5, no. 5, pp. 893-908, 2011

[15] J. Pratt, C.-M. Chew, A. Torres, P. Dilworth, and G. Pratt, "Virtual model control: An intuitive approach for bipedal locomotion," International Journal of Robotics Research (IJRR), vol. 20, no. 2, pp. 129-143, 2001.

[16] C. Sunada, D. Argaez, S. Dubowsky, and C. Mavroidis, "A coordinated Jacobian transpose control for mobile multi-limbed robotic systems," in IEEE International Conference on Robotics and Automation, pp. 19101915, IEEE Comput. Soc. Press, 1994.

[17] S. H. Hyon, J. Hale, and G. Cheng, "Full-Body Compliant HumanHumanoid Interaction: Balancing in the Presence of Unknown External Forces," IEEE Transactions on Robotics, vol. 23, pp. 884-898, 2007.

[18] C. Ott, M. A. Roa, and G. Hirzinger, "Posture and balance control for biped robots based on contact force optimization," in IEEE-RAS International Conference on Humanoid Robots, pp. 26-33, IEEE, 2011.

[19] A. L. Torres, Virtual Model Control of a Hexapod Walking Robot. Bachelor thesis, 1996

[20] S. Coros, Simulated Bipedal Locomotion. PhD thesis, 2010.

[21] C. Gehring, S. Coros, M. Hutter, M. Bloesch, M. A. Hoepflinger, and R. Siegwart, "Control of dynamic gaits for a quadrupedal robot," in IEEE International Conference on Robotics and Automation (ICRA), pp. 3287-3292, 2013.

[22] C. Gehring, S. Coros, M. Hutter, C. Dario Bellicoso, H. Heijnen, R. Diethelm, M. Bloesch, P. Fankhauser, J. Hwangbo, M. Hoepflinger, and R. Siegwart, "Practice Makes Perfect: An Optimization-Based Approach to Controlling Agile Motions for a Quadruped Robot," IEEE Robotics \& Automation Magazine, vol. 23, pp. 34-43, 2016.
[23] M. de Lasa and A. Hertzmann, "Prioritized optimization for task-space control," IEEE/RSJ Intenational Conference on Intelligent Robots and Systems (IROS), pp. 5755-5762, 2009.

[24] L. Sentis and O. Khatib, "Control of Free-Floating Humanoid Robots Through Task Prioritization," in IEEE International Conference on Robotics and Automation (ICRA), pp. 1718-1723, 2005.

[25] O. Kanoun, F. Lamiraux, and P.-B. Wieber, "Kinematic Control of Redundant Manipulators: Generalizing the Task-Priority Framework to Inequality Task," IEEE Transactions on Robotics, vol. 27, pp. 785-792, 2011.

[26] M. Hutter, H. Sommer, C. Gehring, M. Hoepflinger, M. Bloesch, and R. Siegwart, "Quadrupedal locomotion using hierarchical operational space control," The International Journal of Robotics Research (IJRR), vol. 33, pp. 1062-1077, 2014.

[27] T. C. Boaventura, Hydraulic Compliance Control of the Quadruped Robot HyQ. PhD thesis, University of Genoa, Italy and Istituto Italiano di Tecnologia (IIT), 2013.

[28] M. Hutter, P. Leemann, S. Stevsic, A. Michel, D. Jud, M. Hoepflinger, R. Siegwart, R. Figi, C. Caduff, M. Loher, and S. Tagmann, "Towards optimal force distribution for walking excavators," in International Conference on Advanced Robotics (ICAR), (Istanbul, Turkey), pp. 295301, IEEE, 2015.

[29] A. Alleyne and R. Liu, "A simplified approach to force control for electro-hydraulic systems," Control Engineering Practice, vol. 8, pp. 1347-1356, 2000.

[30] S. Tafazoli, Identification of Frictional Effects and Structural Dynamics for Improved Control of Hydraulic Manipulators. $\mathrm{PhD}$ thesis, University of British Columbia, 1997.

[31] S. Tafazoli, S. Salcudean, K. Hashtrudi-Zaad, and P. Lawrence, "Impedance control of a teleoperated excavator," IEEE Transactions on Control Systems Technology, vol. 10, pp. 355-367, 2002.

[32] J. Koivumaki and J. Mattila, "Stability-Guaranteed Force-Sensorless Contact Force/Motion Control of Heavy-Duty Hydraulic Manipulators," IEEE Transactions on Robotics, vol. 31, pp. 918-935, 2015.

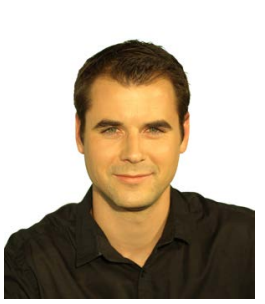

Marco Hutter is Assistant Professor for Robotic Systems at ETH Zurich, Branco Weiss Fellow, and Co-Founder of ANYbotics AG. He studied mechanical engineering and conducted his doctoral degree in robotics at ETH Zurich. Marco Hutter is part of the National Centre of Competence in Research (NCCR) Robotics and NCCR Digital Fabrication. His research interests are in the development of novel machines and actuation concepts, including the underlying control, planning and optimization algorithms for locomotion and manipulation.

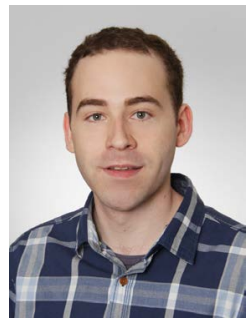

Philipp Leemann received his Bachelor and Master's degree for Mechanical Engineering at ETH Zurich. After completing his Master's Thesis about the automation of a walking excavator in spring 2015, he was employed by ETH Zurich for half a year and is now working as an external Research Assistant at the Robotic Systems Lab (RSL) employed by Moog Inc. 

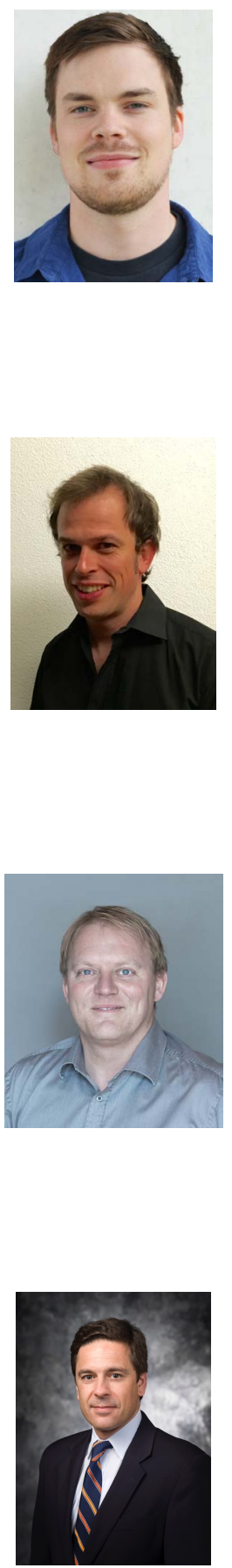

Gonzalo Rey is Chief Technology Officer at Moog Inc., a world wide high performance control systems, equipment and solutions company. He received a Ph.D. from Cornell University where he studied engineering and mathematics. Prior to Moog, he worked at the United Technologies Research Center, and was a Technical Fellow at Pratt and Whitney. His technical interests are in realizing the potential benefits to society of modern robotics and autonomous systems.

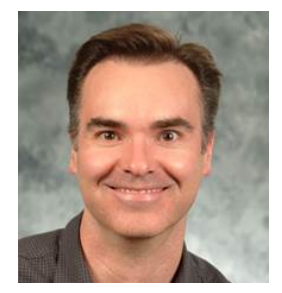

George Small received his Bachelor Degree in Mechanical Engineering in 1986, from the University of Buffalo in New York. He has worked at Moog Inc. over the past 20 years in their aerospace businesses. He has served as Design Engineer, Engineering Manager and Chief Engineer for Flight Controls at Moogs Aircraft Group. For the past 4 years George has served as Principal Engineer at Moog. His present focus areas of interest are in the areas of robotics and autonomy, as well as additive manufacturing. 